\title{
Dirac's Ether in Curved Spacetime-II: The Geometric Amplification of the Cosmic Magnetic Induction
}

\author{
MARCELO CARVALHO ${ }^{1}$, ALEXANDRE L. OLIVEIRA ${ }^{2}$ and CARLOS R. RABAÇA ${ }^{2}$ \\ ${ }^{1}$ Waseda University, Department of Mathematics, 3-4-1 Okubo, Shinjuku-ku, Tokyo 169, Japan \\ ${ }^{2}$ Universidade Federal do Rio de Janeiro, Observatório do Valongo \\ 20080-090 Rio de Janeiro - RJ, Brasil
}

Manuscript received on August 26, 2002; accepted for publication on June 30, 2003;

presented by AFFonso G. Gomes

\begin{abstract}
We search for an amplification mechanism of the seed cosmic magnetic induction by studying a new version of the Dirac's æther in a curved cosmological background. We find that the variation of the scale factor $R(t)$ with cosmic time brings to the magnetic field the desired effect of amplification, that we call geometric amplification.
\end{abstract}

Key words: Dirac's æther, magnetic induction, electromagnetism.

\section{INTRODUCTION}

Information about the universe comes vastly from the propagation of electromagnetic waves throughout the cosmic medium. However, until now the origin of the cosmic magnetic induction (CMI) in astronomical objects remains unknown (see Grasso and Rubinstein 2001 for a comprehensive review). In fact, no theory has completely succeeded in explaining the evolution of the CMI, from its generation in the early universe to the present values found in a multitude of cosmic scales. In stars, magnetic fields range from $10^{8} \mathrm{~T}$ in the interiors of neutron stars, to values of $\approx 1$ $\mathrm{T}$ in sunspots, and down to $10^{-7} \mathrm{~T}$ in protostellar objects. It is generally accepted that in stellar interiors the standard dynamo action, in combination with convective motions and the reconnection of the field lines, is able to explain the range of values observed. In galaxies, however, magnetic dynamos leave many unanswered questions (Parker 1979), the most immediate of them is the rather long characteristic time-scales over which they could operate. For instance, the interstellar medium in our Galaxy has an ordered field of $\approx 2 \times 10^{-10} \mathrm{~T}$, superposed upon which there is a random

Correspondence to: Alexandre L. Oliveira

E-mail: alexandr@ov.ufrj.br 
component of $\approx 1-2$ times this value (Taylor and Cordes 1993). Considering that the period of rotation of the interstellar gas about the Galactic center is $\approx 2.5 \times 10^{8}$ years, there would have been at most 50 complete rotations of this gas about the center. The differential rotation of the ionized gas in the interstellar medium results in the stretching and amplification of the magnetic field in the disk. Therefore, any primordial magnetic field would be tightly wound up. The problem is that this mechanism is not enough to produce an ordered field. The winding up of the field lines would result in tightly wound tubes of magnetic flux running in opposite directions. It is then necessary that a mechanism exists that is able to reconnect the lines of force in order to create the large uniform field.

As we proceed to even larger structures, evidence for large scale magnetic fields are seen. In the intracluster medium in cluster of galaxies, fields of $\approx 10^{-10} \mathrm{~T}$ have been derived from the diffuse synchrotron radio emission observed from a number of clusters of galaxies, as well as from the observation of depolarization of the emission of extended radio sources by the surrounding medium. In the intergalactic medium between clusters of galaxies, we are only able to set an upper limit for the magnetic field of $10^{-13} \mathrm{~T}$; a value provided from the lack of polarization of the emission reaching us from distant radio sources. However, it is widely believed that magnetic inductions are present in the universe whatever the scale we look for, and new technological developments are in their way to confirm this (e.g., the SIRTF infrared satellite and the SOAR optical telescope). Whether present-day field values were built up when the first galaxies formed remains questionable, but even if this is the case, the process requires the existence of a very weak seed field that was slowly amplified over cosmic time.

It has been shown that it is possible to generate a seed CMI in a plasma with no fields present at the recombination time supposing that there are only variations in the pressure of the electrons in the plasma. This effect is known as the Biermann battery (see Parker 1979). The flow of electrons to lower pressure regions results in a charged unbalanced plasma, which produces an electric field opposing the flow of electrons. As a result, the flow stops and an electric and magnetic field (emf) is created. This emf cannot drive a current though, since the integral around any closed loop in the case of a linear gradient is zero. If, however, there are variations in the electron density throughout the plasma, different emfs can be induced in different regions and then currents flow in the plasma creating a magnetic field. Nevertheless, this process saturates at about $10^{-25} \mathrm{~T}$, since it is limited by the self-inductance of the current loop itself. How then to reconcile this extremely low value with the upper limit found today for the intergalactic medium between clusters?

Recently, one special mechanism has been studied in which the amplification of a seed CMI is understood as being caused by the expansion of the cosmological background (Oliveira 2001, see also references therein). This is called geometric amplification, because the only agent responsible is the geometric scale factor $R(t)$. Advantages of this approach are: i) it does not rule out the existence of other mechanisms of amplification, ii) the amplification factor obtained for the seed CMI is kept within observational constraints. 
In a previous paper, Oliveira and Teixeira (2000) studied the equations of a Dirac's æther (Dirac 1951a, b) coupled to a Proca field in the background of an Einstein static universe. In this paper we search for a geometric amplification of the seed CMI by applying to the cosmic medium a recent version of the Dirac's æther model (Carvalho and Oliveira 2003). This new version of the Dirac's æther model maintains the most important features of the original Dirac's model, but adds a new interpretation of the 4-velocity: it is the velocity of the different parts of the æther relative to a generic observer, inertial or not.

This work is organized as follows. In Section $\mathbf{2}$ we describe the model and justify the adoption of a Dirac framework with a very small conductivity term. In Section $\mathbf{3}$ we write down the equations for the proposed field in a curved background. In Section 4 we present numerical solutions to the problem and study their stability. In the last section we devote ourselves to concluding remarks.

\section{THE MODEL}

At a local level, Maxwell's theory is the best we have to explain most of the occurring electromagnetic phenomena. In general, the validity of the theory is taken for granted up to cosmic scales, but many authors have pointed out that some of the behaviors of the electromagnetic field in a supposedly curved background are better explained by alternative theories. In fact, since Einstein's general relativity theory it is known that a curved background brings to a propagating photon a sort of effective mass (Crawford 1979) or, in other words, a finite range behavior to the electromagnetic field. This is the main reason why finite range theories cannot be ruled out as an alternative explanation for the electromagnetic field at cosmic scales. The finite range theory of electromagnetism is called Proca's theory (Goldhaber and Nieto 1971), which corresponds to, after quantization, a non-null photon rest mass.

A relationship is known (Vigier 1990) between the photon rest mass $m_{\gamma}$ (or the corresponding range of the Proca's theory) and the conductivity $\sigma$ of the Dirac's æther, $m_{\gamma}=\sigma h / c$, where $h$ is the Planck's constant and $c$ is the speed of light. Supposing that the æther conductivy is $\approx 10^{-13}$ $/ \mathrm{s}$, it corresponds to a photon mass of $\approx 10^{-48} \mathrm{~g}$, which lies below current laboratory estimates. Therefore, the magnetohydrodynamic equations do not apply to our problem, since they presume the existence of a highly conductive medium (Alfvén and Falthammar 1963). We will assume a minimal interchange between electromagnetic and gravitational fields in the curved space-time, and apply the usual equations of the electromagnetic theory in the presence of a curved background.

The proposed action has a self interaction term, and is expressed by

$$
\mathcal{S}=\int d x\left(-\frac{1}{4} F^{2}+\sigma v_{\alpha} F^{\alpha \mu} A_{\mu}\right)
$$

where $A^{\mu}$ is the electromagnetic 4-potential, $F_{\alpha \mu}=\partial_{\alpha} A_{\mu}-\partial_{\mu} A_{\alpha}$ are the components of the electromagnetic field tensor, and $v_{\alpha}$ is the velocity of a generic observer, inertial or not.

The equations of motion for $A_{\mu}$ in a flat space-time are

$$
\partial_{\nu} F^{\nu \mu}+\sigma v^{\mu} \partial . A-\sigma v_{\nu} \partial^{\mu} A^{\nu}=0 .
$$


It is implicit to our formulation that an inertial observer moving with the cosmic æther has velocity components $v_{æ \text { ther }}^{\mu}=(1 ; 0,0,0)$.

\section{EQUATIONS FOR A CURVED BACKGROUND}

The equations (1) for an electromagnetic field that is minimally coupled with the geometry are (Carvalho and Oliveira 2003)

$$
F_{; \mu}^{\mu v}+\frac{\sigma}{c}\left(A^{\mu} v^{v}-A^{v} v^{\mu}\right)_{; \mu}=J^{v}
$$

where the semicolon denotes the covariant derivative, and $J^{\nu} \equiv(-\sigma / c) v_{\mu} F^{\mu \nu}$. Equations (2) differ from the ones in Oliveira and Teixeira (2000) by the presence of the skew-symmetric term $A^{\mu} v^{v}-A^{v} v^{\mu}$ instead of the term $\left(1 / \lambda^{2}\right) A^{v}$ (that comes from Proca's theory). We adopt a cylindrical coordinate system $x^{\mu}=(t ; \rho, \phi, \zeta)$ in a Friedmann cosmological background. The metric tensor in all three Friedmann geometries and the field $A^{\mu}$, with cylindrical symmetry, are given by

$$
\begin{aligned}
g_{\mu \nu} & =R^{2}(t) \operatorname{diag}\left[\left(1 ;-1,-u^{2}(\rho),-w^{2}(\rho)\right)\right], \\
A^{\mu} & =(0 ; 0,1,0) f(t) / R^{2}(t) .
\end{aligned}
$$

where $u(\rho)$ and $w(\rho)$ are functions of the coordinate $\rho$; the conformal time $t$ is defined by $t=c t^{\prime} / R$, where $t^{\prime}$ is the usual cosmic time; and $R$ is the scale factor. Depending on the geometry of the spacetime, they will define the type of three-geometry (with constant curvature $k_{c}$ ) under consideration. $f(t)$ is a function to be determined by the field equations. The velocity of observers moving with the cosmic æther is $v_{\mu}=R(t) \delta_{\mu}^{0}$.

The field strength $F_{\mu \nu}$ has non-zero independent components $F_{02}$ and $F_{12}$. In an orthonormal basis, the non-null components of the fields $\mathbf{E}$ and $c \mathbf{B}$ are

$$
E_{\phi}=-\dot{f} u^{2} / R^{2}, \quad c B_{\zeta}=2 f u u^{\prime} / R^{2}
$$

where the dot means $d / d t$, and the prime is $d / d \rho$. The electric field and the magnetic induction are orthogonal and non-homogeneous, and both depend on $t$ and $\rho$. Their moduli are

$$
|\mathbf{E}|=|u \dot{f}| / R^{2}, \quad \quad|\mathbf{B}|=2\left|f u^{\prime}\right| /\left(c R^{2}\right) .
$$

Table I shows the curvature $k_{c}$ of each one of the Friedmann's geometries considered, $u(\rho), w(\rho)$, $R(t)$, the Hubble constant (or Hubble parameter, constant at every point in the 3-space, at a given time), the density parameter, and the equation for $f(t)$ that needs to be solved. The curvature of the Friedmann models used, compatible with the scale factor, is generated by a perfect fluid (see Zel'dovich and Novikov 1983) with zero pressure (dust).

We are particularly interested in finding solutions for the CMI that provide an explanation for the amplification of the magnetic induction below the suggested limit of $10^{-13} \mathrm{~T}$ for the intergalactic medium between clusters of galaxies. 
TABLE I

Curvature $k_{c}, u(\rho), w(\rho), R(t)$, and equations to be solved.

\begin{tabular}{ccccccc}
\hline$k_{c}$ & $u(\rho)$ & $w(\rho)$ & $R(t)$ & Hubble Constant & $\Omega(t)$ & Equation for $f(t)$ \\
\hline \hline 0 & $\rho$ & 1 & $(\alpha / 2) t^{2}$ & $\alpha^{-1} 4 c t^{-3}=\frac{2}{3} t^{\prime-1}$ & 1 & $\ddot{f}-\alpha t(\sigma / c) f=0$ \\
\hline+1 & $\sin \rho$ & $\cos \rho$ & $\alpha(1-\cos t)$ & $\alpha^{-1} c \sin t(1-\cos t)^{-2}$ & $2(1+\cos t)^{-1}$ & $\ddot{f}+[4-(\sigma / c) \alpha \sin t] f=0$ \\
\hline-1 & $\sinh \rho$ & $\cosh \rho$ & $\alpha(\cosh t-1)$ & $\alpha^{-1} c \sinh t(\cosh t-1)^{-2}$ & $2(\cosh t+1)^{-1}$ & $\ddot{f}-[4+(\sigma / c) \alpha \sinh t] f=0$ \\
\hline
\end{tabular}

\section{SOLUTIONS}

For now on, we assume that the conductivity of the Dirac's æther is $10^{-19} \mathrm{~s}^{-1}$, and the initial CMI is $10^{-25} \mathrm{~T}$. Our model also includes a weak initial electric field of magnitude $10^{-4} \mathrm{~V} / \mathrm{m}$ to be dissipated during the time evolution. These limits are fixed in order to provide a realistic value for the modulus of the CMI that agrees with the one established by the usual theory of the cosmic fields. The values adopted should not perturb the gravitational field; from a simple calculation it is evident that the energy-momentum tensor of the electromagnetic and gravitational fields are related by a factor above $10^{10}$.

The third equation for $f(t)$ given in the last column of Table I requires a numerical solution, and therefore we initially chose to integrate numerically all three equations. In order to perform the numerical integration, we used the adimensional conformal cosmic time as defined in the previous section. The initial and final times adopted are $t_{i}=0.0890$ and $t_{f}=1.6100$. These values were obtained by assuming a value of $100 \mathrm{~km} \mathrm{~s}^{-1} \mathrm{Mpc}^{-1}$ for the present day Hubble constant in a flat universe $\left(\Omega_{0}=1\right)$, and $\alpha$ is $8.87 \times 10^{25} \mathrm{~m}$. For the other $\Omega_{0} \neq 1$ models, the present value of the Hubble constant changes very little. In the standard cosmology, $t_{i}$ and $t_{f}$ correspond, respectively, to the final stage of the matter-radiation coupling and to our current epoch. We used two different integration steps: $\Delta t=10^{-11}$ for regions where the fields are more sensitive to the variations in $t$, and $\Delta t=10^{-4}$ for the other regions; about 20, 000 steps are performed. In Table II, we display a small ensemble of points in order to show the amplification phenomenon in terms of the quantities $\mathcal{E}(t) \equiv|\mathbf{E} / u|=|\dot{f}| / R^{2}$ and $\mathcal{B}(t) \equiv\left|\mathbf{B} / u^{\prime}\right|=2|f| /\left(c R^{2}\right)$, that for simplicity we will also refer to as the electric field and magnetic induction. Comparing the initial and final values of the fields for each geometry, the data show an amplification of the magnetic induction of the order of $\approx 10^{+6}$, and an overall reduction of the electric field of the order of $\approx 10^{-5}$.

It is interesting to note some points about the equations for $f(t)$ in Table I. The first equation (for $k_{c}=0$ ) has an analytical solution of the form

$$
f(t)=C_{1} A\left(-30^{-1 / 3} t\right)+C_{2} B\left(-30^{-1 / 3} t\right)
$$

where $A$ and $B$ are two linearly independent, modified Bessel functions, known respectively as 
TABLE II

Some Results of the Numerical Integration.

\begin{tabular}{c|c|c|c|c|c|c}
\hline \multirow{2}{*}{$t$} & \multicolumn{2}{|c|}{ Flat $\left(k_{c}=0\right)$} & \multicolumn{2}{c}{ Elliptic $\left(k_{c}=+1\right)$} & \multicolumn{2}{c}{ Hyperbolic $\left(k_{c}=-1\right)$} \\
\cline { 2 - 7 } & $\log |\mathcal{E}|$ & $\log |c \mathcal{B}|$ & $\log |\mathcal{E}|$ & $\log |c \mathcal{B}|$ & $\log |\mathcal{E}|$ & $\log |c \mathcal{B}|$ \\
\hline \hline 0.0890 & -4.0004 & -14.6999 & -3.9998 & -14.6988 & -4.0009 & -14.6999 \\
\hline 0.1000 & -4.2028 & -5.8604 & -4.2021 & -5.8597 & -4.2036 & -5.8611 \\
\hline 0.2000 & -5.4069 & -6.0606 & -5.4040 & -6.0577 & -5.4098 & -6.0635 \\
\hline 0.3000 & -6.1112 & -6.4859 & -6.1048 & -6.4795 & -6.1178 & -6.4925 \\
\hline 0.5000 & -6.9986 & -7.0838 & -6.9807 & -7.0657 & -7.0166 & -7.1018 \\
\hline 0.8000 & -7.8147 & -7.6621 & -7.7690 & -7.6158 & -7.8607 & -7.708 \\
\hline 1.0000 & -8.2018 & -7.9419 & -8.1308 & -7.8695 & -8.2734 & -8.0138 \\
\hline 1.2000 & -8.5177 & -8.1723 & -8.4156 & -8.0678 & -8.6204 & -8.2753 \\
\hline 1.5000 & -8.9034 & -8.4557 & -8.7441 & -8.2913 & -9.0624 & -8.6154 \\
\hline 1.6000 & -9.0147 & -8.5378 & -8.8333 & -8.3505 & -9.1049 & -8.7191 \\
\hline
\end{tabular}

even and odd Airy functions. Constants $C_{1}$ and $C_{2}$ determined from the initial conditions are

$$
C 1 \approx-0.22198 \times 10^{45}, \quad C 2 \approx+0.36876 \times 10^{45} .
$$

The existence of the analytical solution provides a way to check the quality of our numerical integration. As can be seen in Figure 1, the agreement between the solid line (analytical solution) and the points (numerical solution) is very good, giving us confidence in our results. The dashed lines for $t>t_{f}$ represent the long term behavior of the fields, presented here in order to show that even though the amplification of the CMI tend to decrease after an early peak, it is still preserved. The point here is that a small initial decrease of the electric field induces a fairly large initial amplification of the CMI. This behavior is also predicted by the usual Maxwellian theory, in which the decrease of one field induces the enlargement of the other. After a few steps, both fields tend to converge to similar values, and keep diminishing as the universe expands. A more detailed study of similar cases can be found in Oliveira (2001). Even after a long conformal time, e.g. $t=3.0$, the amplification effect observed is as large as $10^{4}$, therefore very meaningful to the CMI.

The second equation in Table I (for $k_{c}=+1$ ) can be rewritten as:

$$
\frac{d^{2} f}{d z^{2}}+[a-2 q \cos 2 z] f=0,
$$

where $a=16$ and $q=2(\sigma / c) \alpha=2 / 30$ and $2 z=\pi / 2-t$. This is a typical Mathieu equation. One important question to consider is the stability of the solutions. They can be either unstable (solutions that grow exponentially as t increases) or stable (solutions that remain bound), depending on the combination of the parameters $a$ and $q$. Since $a$ is fixed and positive, it can be observed at 


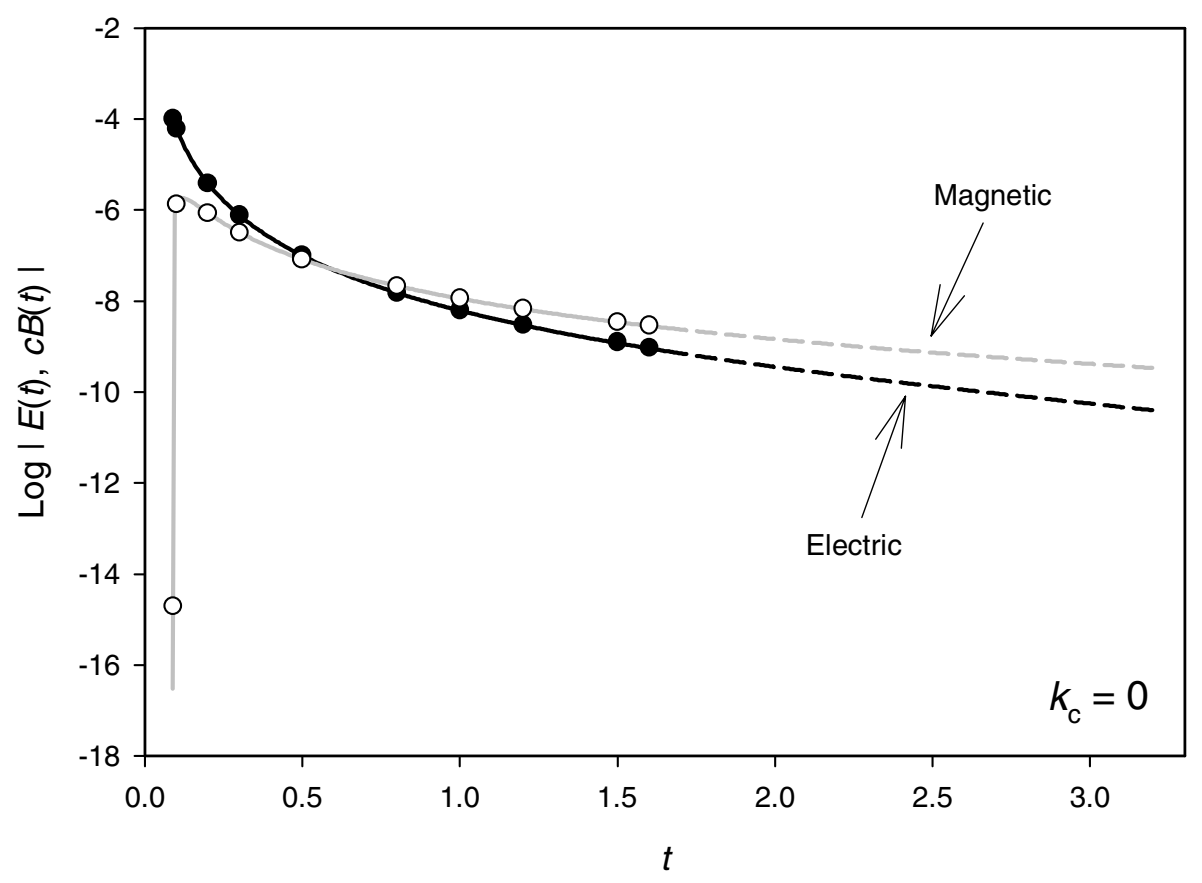

Fig. 1 - The graphic shows a comparison between the analytical and the numerical solutions for the fields in the $k_{c}=0$ universe. A description of the symbols is given in the text.

once that if $q$ were zero the equation would be that of a simple-harmonic motion. Combinations of $a$ and $q$ lying in the range $|2 q|<a$ might be expected to lead to essencially stable solutions. However, the values adopted here for both parameters place the system in a limiar regime of instability. In order to check the behavior or our equation, we used the numerical code CHARMA (Leeb 1979), that calculates characteristic values $a$ of the Mathieu's differential equation, given $q$, for solutions of $f(t)$ of periodicity $\pi$ or $2 \pi$. The derived values of $a$ are correct to at least 9 decimal places. After a quick transformation of our equation to the one presented in Leeb, we found that the solution of our equation is stable, physically meaning that no resonance is present. Therefore, the obtained effect of CMI amplification is not expected to change significantly over a larger integration period.

It should be noted that the results presented are determined not only by the evolution of the function $f(t)$ (which constrains the field equations) but also by the direct contribution of the geometry as given by the scale factor, $R(t)$, that is present in both mathematical expressions for $\mathcal{E}$ and $\mathcal{B}$. It is the interchange between the gravitational and the electromagnetic fields that imposes, as the Universe evolves, the decrease of the electric field and the amplification of the CMI.

\section{CONCLUSION}

In the new version of the Dirac's æther studied here the cosmic medium is described in the context of a curved background in which a tiny conductivity term is present. Our results show an amplification 
of the initial CMI together with a reduction of the electric field. The model does not contradict the present day constraints on the effective mass of a photon, and has the advantage of incorporating in a natural way the 4-velocity (first pointed out by Dirac 1951a, b), which is interpreted as the velocity of the æther with respect to any observer, inertial or not: an observer with $v_{\alpha}=R(t) \delta_{\alpha}^{0}$ sees the same phenomenon of amplification of the CMI and reduction of the electric field. The same amplification phenomenon had already been observed by Oliveira (2001) in the context of a Proca's electromagnetic field in a Dirac's æther.

The geometric relations between the electromagnetic field and the metric tensor involve the scale factor $R(t)$. The amplification of the CMI in our model is determined by the coupling between the electromagnetic and the gravitational fields as in the usual theory of electromagnetism in a curved background. Larger couplings between both fields will lead to an even faster and/or more intense interchange, as can be seen in Turner and Widrow (1988). In the same way that the geometric amplification describes how the expansion of the universe influences the electromagnetic field, there is also the possibility that the electromagnetic field influences the expansion of the universe (Matravers and Tsagas 2000). Our results are compatible with a strict relationship between both phenomena.

At last, it is important to stress that the effect of geometric amplification described here absolutely does not replace other mechanisms of production of large uniform fields, as the conventional dynamo effect, believed to be present in the interior of stars, intragalactic medium, and even at the scale of galaxies. We do believe, however, that at cosmic scales, where the dynamo action most probably fails, the geometric amplification of the seed CMI may be an important effect to be taken into consideration. In a near future technological advances will be able to detect these fields and confirm our results.

\section{ACKNOWLEDGMENTS}

A. L. Oliveira thanks the Centro Brasileiro de Pesquisas Físicas (CBPF) for support. M. Carvalho thanks Japanese monbusho for the scholarship, Prof. T. Kori for helpfull discussions, and acknowledges the constant support from Aurelina Carvalho, Jose E. Carvalho, Ying Chen, IC XC Nika. All of us thank J. A. Helayel-Neto and A. F. F. Teixeira (CBPF) for his friendly encouragement. At last but not least, we thank the anonymous referee for his careful reading of the manuscript, and for the innumerous contributions given.

\section{RESUMO}

Procuramos por um mecanismo de amplificação das sementes do campo de indução magnética cósmico através do estudo de uma nova versão do æther de Dirac em uma arena cosmológica curva. Encontramos uma amplificação que ocorre se o fator de escala $R(t)$ varia com o tempo cósmico, acarretando um efeito o qual chamamos de amplificação geométrica do campo magnético.

Palavras-chave: Æther de Dirac, indução magnética, eletromagnetismo. 


\section{REFERENCES}

Alfvén H and Falthammar C-G. 1963. Cosmical Electrodynamics. Oxford University Press.

Carvalho M and Oliveira AL. 2003. A New Version of Dirac's Æther and Its Cosmological Applications, Found Phys Lett 16: 255-263, hep-th/0212319.

CRAWFord DF. 1979. Photon decay in curved space-time Nature 277: 633-635.

DiRAC PAM. 1951a. Is There an Æther. Nature 168: 906-907.

Dirac PAM. 1951b. A new classical theory of electrons. Proc Roy Soc A 209: 291-296.

Goldhaber AS and Nieto MM. 1971. Terrestrial and Extraterrestrial Limits on The Photon Mass. Rev Mod Phys 43: 277-296.

Grasso D and Rubinstein HR. 2001. Magnetic Fields in the Early Universe. Phys Rep 348: 163-266.

LEEB WR. 1979. Characteristic Values of Mathieu's Differential Equation, ACM Transactions on Mathematical Software 5: 112-117.

Matravers DR and Tsagas CG. 2000. Cosmic magnetism, curvature, and the expansion dynamics. Phys Rev D 62: 103519(1)-103519(7).

Oliveira AL. 2001. On the Amplification of the Cosmological Non-Maxwellian Fields in Curved Background. Mod Phys Lett A 16: 541-555.

Oliveira AL and Teixeira AFF. 2000. Dirac's æther in curved spacetime. An Acad Bras Cienc 72: 161-164. See also the references therein (Oliveira 2001).

PArker EN. 1979. Cosmical Magnetic Fields, Claredon Press.

TAYlor J AND CORdes JM. 1993. Pulsar distances and the galactic distribution of free electrons. Ap J 411: $674-684$.

Turner MS AND Widrow LM. 1988. Inflation-produced, large-scale magnetic fields. Phys Rev D 37: 2743-2754.

VIGIER JP. 1990. Evidence For Nonzero Mass Photons Associated With a Vacuum-Induced Dissipative Red-Shift Mechanism. IEEE Transactions on Plasma Science 18: 64-72.

ZeL'Dovich YaB and Novikov ID. 1983. The Structure and Evolution of the Universe. The University Chicago Press. 